\title{
Identification and Analysis of Chemical Constituents and Rat Serum Metabolites in Lycopodium clavatum Using UPLC-Q-TOF/MS Combined with Multiple Data-Processing Approaches
}

\author{
Xin Li, ${ }^{1}$ Mingqin Kang, ${ }^{2}$ Ningning Ma, ${ }^{1}$ Tan Pang, ${ }^{3}$ Yanjun Zhang $\left(D,{ }^{4}\right.$ Hua Jin, ${ }^{5}$ \\ Zhen Yang $\mathbb{1},{ }^{1}$ and Lili Song $\mathbb{1}^{1}$ \\ ${ }^{1}$ School of Chinese Materia Medica, Tianjin University of Traditional Chinese Medicine, Jian Kang Chan Ye Yuan, Jinghai Dist., \\ Tianjin 301617, China \\ ${ }^{2}$ Changchun Customs (Former Jilin Inspection and Quarantine Bureau), Changchun 130062, China \\ ${ }^{3}$ Pharmaron (Beijing) Inc., Beijing 100176, China \\ ${ }^{4}$ Tianjin State Key Laboratory of Modern Chinese Medicine, Tianjin Key Laboratory of Traditional Chinese \\ Medicine Pharmacology, Tianjin Key Laboratory of TCM Chemistry and Analysis, Tianjin University of Traditional \\ Chinese Medicine, Jian Kang Chan Ye Yuan, Jinghai Dist., Tianjin 301617, China \\ ${ }^{5}$ College of Traditional Chinese Medicine, Tianjin University of Traditional Chinese Medicine, Jian Kang Chan Ye Yuan, \\ Jinghai Dist., Tianjin 301617, China
}

Correspondence should be addressed to Zhen Yang; yzwygb@126.com and Lili Song; sll0204@163.com

Xin Li and Mingqin Kang contributed equally to this work.

Received 16 April 2019; Accepted 2 June 2019; Published 2 July 2019

Academic Editor: Mohammed S. Ali-Shtayeh

Copyright (C) 2019 Xin Li et al. This is an open access article distributed under the Creative Commons Attribution License, which permits unrestricted use, distribution, and reproduction in any medium, provided the original work is properly cited.

Lycopodium clavatum is a dry whole grass of Lycopodium japonicum Thunb.; it has been extensively used to anti-inflammatory, antioxidant, and antimicrobial actions and inhibits acetylcholinesterase activity. However, it lacks further compounds research of Lycopodium clavatum in vivo and in vitro. In this work, a rapid method was established using the ultra high performance liquid chromatography with quadrupole-time-of-flight mass spectrometry (UPLC-Q-TOF/MS) combined with multiple data-processing approaches for compounds analysis of Lycopodium clavatum in vitro and in vivo. Finally, 30 peaks were characterized in $75 \%$ ethanol extract of Lycopodium clavatum and 17 peaks were characterized in rat plasma that including 12 prototype compounds and 5 metabolites. Methylation and demethylation are the main transformation reactions of Lycopodium clavatum in rat serum. This work could be helpful for understanding the complex compounds of Lycopodium clavatum and further analyzing the pharmacological studies of active compounds.

\section{Introduction}

Lycopodium clavatum (LC) is a dry whole grass of Lycopodium japonicum Thunb.; it has been widely used to antiinflammatory [1], antioxidant, and antimicrobial actions [2] and inhibits acetylcholinesterase activity [3-5], meanwhile, it has antioxidative, antiproliferative, and biochemical effects for HepG2 cells [6]. In recent years, literature reports of LC pay more attention to analyzing component in vitro.
LC mainly contains alkaloids [7] and triterpenoids [8-11]. Alkaloids are divided into four types with the structural characteristics, including lycopodine, lycodine, fawcettimine, and miscellaneous. Four types of mother nucleus all have the same configuration, so it is inferred that there is a common biological origin and transformation relationship between them $[12,13]$. So far, there have been few reports on the chemical constituents of LC, and there is almost no research on the blood components in the body. Therefore, 
it is especially important for the chemical composition of LC in vivo and in vitro. At present, LC-MS is often used to analyze and determine the component of traditional Chinese medicine, especially the technology of UPLC-Q-TOF/MS $[14,15]$. UPLC-Q-TOF/MS combined with multiple data processing approaches can improve efficiency and obtain abundant information, which can be utilized as a practical model for the study of component $[16,17]$. The combination of the technology has the advantages of high efficiency, wide molecular mass range, high sensitivity, low error rate, and high reproducibility $[18,19]$. In this work, a rapid method will be established for the compounds analysis of LC in vitro and in vivo by the ultra high performance liquid chromatography (UPLC) with quadrupole-time-of-flight mass spectrometry combined with multiple data-processing approaches, which can be used to further analyze the chemical composition in vivo and in vitro.

\section{Methods and Materials}

2.1. Chemicals and Materials. Lycopodium clavatum was purchased from Beijing Huamiao Pharmaceutical Co. Ltd. It was identified as a dry whole grass of Lycopodium japonicum Thunb. by Professor Tianxiang Li from the College of Traditional Chinese Medicine of Tianjin University of Traditional Chinese Medicine. Methanol and acetonitrile (HPLC grade) were obtained from Sigma-Aldrich (America). Formic acid (HPLC grade) was purchased from Aladdin (America). Water was acquired from Wahaha (China). Ethanol (analysis grade) was purchased from Beijing Chemical Works (China). Male SD rats $(200 \pm 10 \mathrm{~g})$ were obtained from Beijing Huakangkang Biotechnology Co. Ltd.

2.2. Preparation of Lycopodium clavatum Samples In Vitro. Lycopodium clavatum were added 10 times and 6 times $75 \%$ $(v / v)$ ethanol, respectively, and were reflux extracted twice for $1 \mathrm{~h}$ each time. After being extracted, the extract was filtered and condensed to $1 \mathrm{~g} / \mathrm{mL}$. The concentrate was diluted to $10 \mathrm{mg} / \mathrm{mL}$ by water with acetonitrile: $0.1 \%$ formic acid $=1: 1$ $(v / v)$ and through $0.22 \mu \mathrm{m}$ filter membranes before analysis.

2.3. Animal Experiments. Male SD rats were acclimatized for 1 week before the experiment and collected blank blood. The suspension solution of Lycopodium clavatum was intragastri$\mathrm{cal}$ administered at a dose of $10.8 \mathrm{~g} / \mathrm{kg}$ for 3 days. After the last administration, the rats were collected blood by orbital vein at $15 \mathrm{~min}, 1 \mathrm{~h}, 4 \mathrm{~h}$, and $8 \mathrm{~h}$. All samples were centrifuged at $3,000 \mathrm{rpm}$ for $15 \mathrm{~min}$ at $4^{\circ} \mathrm{C}$ and the plasma supernatant was pipetted.

2.4. Preparation of Plasma Samples In Vivo. For analysis, plasma was consolidated including the different blood collection points and $500 \mu \mathrm{L}$ plasma was added $1 \mathrm{~mL}$ acetonitrile for protein precipitation. After vortexed vigorously for $1 \mathrm{~min}$, the samples were centrifuged at $13,000 \mathrm{rpm}$ for $15 \mathrm{~min}$ at $4^{\circ} \mathrm{C}$ and pipetted plasma supernatant. $1 \mathrm{~mL}$ acetonitrile was added to the residue and the above operation was repeated one time. Two-part supernatants were combined and was evaporated to dried under $\mathrm{N}_{2}$ at $45^{\circ} \mathrm{C}$. The residue was dissolved with $100 \mu \mathrm{L}$ methanol, vortex-mixed for $1 \mathrm{~min}$ before being centrifuged at $13,000 \mathrm{rpm}$ at $4^{\circ} \mathrm{C}$ for $15 \mathrm{~min}$ and then pipetted plasma supernatant for analysis.
2.5. Chromatographic Analysis. The supernatant of sample was using A Waters ACQUITY I Class UPLC coupled to a Waters Xevo G2 Q TOF mass spectrometer, where the parameters were as follows. The chromatographic column was ACQUITY UPLC HSS T3 Column $(2.1 \times 100$ mm, $1.8 \mu \mathrm{m}$, Waters Corporation, America). Mobile phases consisted of acetonitrile with $0.1 \%$ formic acid (phase A) and ultrapure water with $0.1 \%$ formic acid (phase B). Whole analysis process, runtime was $25 \mathrm{~min}$, the flow rate was set at 0.2 $\mathrm{mL} / \mathrm{min}$, and the temperature of the column was set at $40^{\circ} \mathrm{C}$. The linear elution gradient program was used as follows: $0.5-$ $1.5 \mathrm{~min}, 99-90 \% \mathrm{~B} ; 1.5-4 \mathrm{~min}, 90-85 \% \mathrm{~B}$; 4-8 min, $85-70 \% \mathrm{~B}$; 8-9 $\min , 70-50 \%$ B; 9-11.5min, 50-20\% B; 11.5-14 min, 20-0\% B; $14-17 \mathrm{~min}, 0 \% \mathrm{~B} ; 17-18 \mathrm{~min}, 0-99 \% \mathrm{~B}$. The injection volume was set at $5 \mu \mathrm{L}$.

2.6. MS Conditions. An UPLC-Q-TOF/MS (waters, America) instrument is equipped with both positive and negative ESI source for sample analysis. The samples were measured from 50 to 1,000 mass-to-charge ratios $(\mathrm{m} / \mathrm{z})$ and the temperature of nitrogen was set to $350^{\circ} \mathrm{C}$. The temperature of dissolvent was set to $80^{\circ} \mathrm{C}$ and the gas flow was set to $600 \mathrm{~L} / \mathrm{h}$. The capillary voltage was set to $3.0 \mathrm{kV}$ in positive ion mode, and it was set to $2.5 \mathrm{kV}$ in negative ion mode.

2.7. Data Processing. A rapid analysis of the extract of Lycopodium clavatum was carried out to obtain the retention time and the exact molecular weight of each component. The possible elemental composition was obtained through the MassLynx V4.1 workstation, and only those formulas with an error less than $10 \mathrm{ppm}$ were accepted. The probable molecular formulas were matched with the relevant literature and databases such as SciFinder and MassBank. Meanwhile, to combine with MS and MS/MS information inferred the compound and its structure.

Raw spectrometric data were processed by MassLynx software (version 4.1, Waters Co., Cicero, America) and converted to a data matrix contain sample name, $\mathrm{t}-\mathrm{m} / \mathrm{z}$ and peak intensity. Data was further processed, including peak matching, peak alignment, and normalization, before multivariate analysis. Multivariate analysis was performed using a combination of principal component analysis (PCA) and the orthogonal projection to least-squared discriminant analysis (OPLS-DA) by SIMCA-P software (version 12.0), and then was further performed using $t$-test for Microsoft Excel software (version 2010). Metabolites were retained in the model based on a combination of variable importance in projection (VIP) greater than 1.0 and $\mathrm{P}$ value of less than 0.05 .

MetaboLynx XS version 4.1 (Waters, Milford, America) can be used to find the unknown drug metabolites in vivo. Biotransformation reactions were set according to its different structure and the peak intensity threshold of 40 with tolerance was set at $0.05 \mathrm{Da}$. The result of possible metabolites and its biotransformation reactions were determined by the relevant literature and MS and MS/MS information.

\section{Results and Discussion}

3.1. Establishment of Compounds Database. To quickly and easily identify the compounds of Lycopodium clavatum, we 


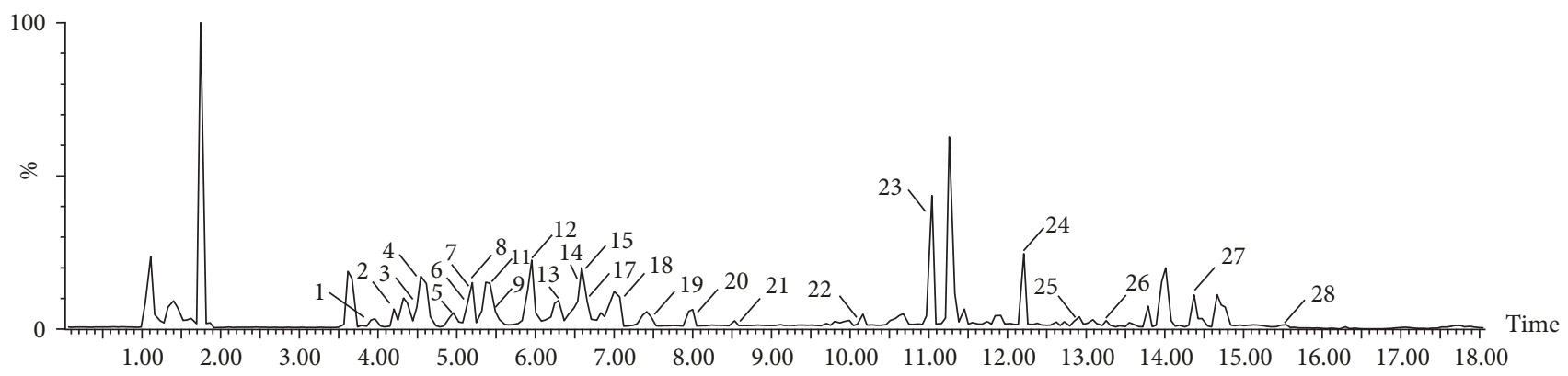

(a)

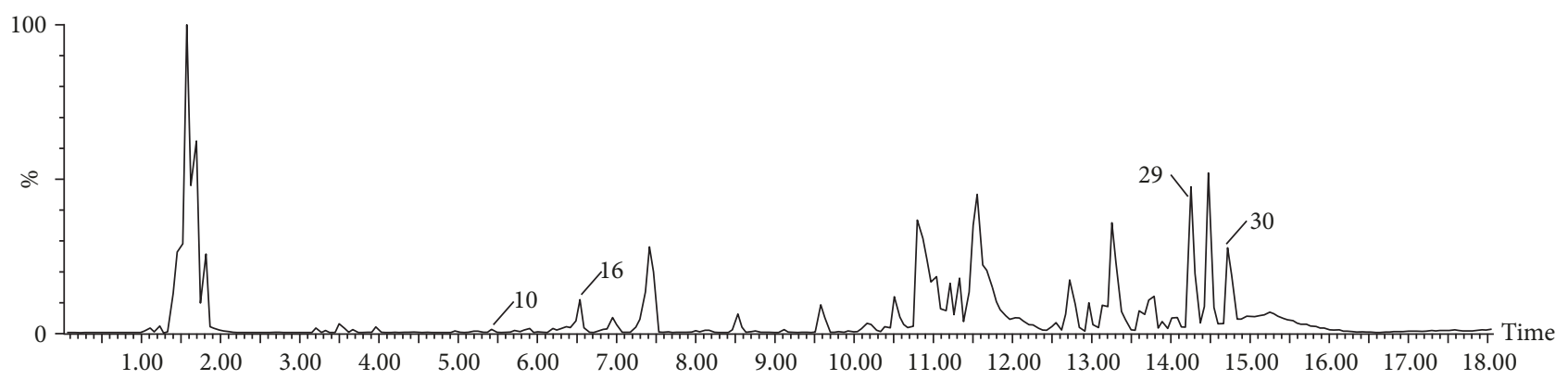

(b)

FIGURE 1: ESI base peak ion (BPI) chromatogram of the extract sample of Lycopodium clavatum extracts analyzed by UPLC-Q-TOF/MS in both positive (a) and negative (b) ion mode.

established compounds database by collecting information of literature in ten years [7-11, 20-24] and searching web databases including Pubmed, SciFinder, and MassBank. The database includes 121 prototype compounds of Lycopodium clavatum that was shown in Table S1, including name, molecular formula, molecular weight, and structure, to support the results of identification for further analysis in vitro and in vivo.

3.2. UHPLC-MS Analysis and Identification of Lycopodium clavatum In Vitro. Under the above MS and chromatographic conditions, the chromatogram of the base peak ion (BPI), the extract sample of Lycopodium clavatum was shown in Figure 1. According to the chromatogram, the baseline was stable and the chromatographic peaks were well separated, indicating that the various components of Lycopodium clavatum extract could be well separated in the data collection range.

The potential compounds were identified by comparing the information of MS and MS/MS; meanwhile, the result was associated with the relevant literature and databases for further determination. For Examples, the precise molecular weight was 366.2274 and the retention time was $7.89 \mathrm{~min}$. The main fragment ions that were analyzed by the MS/MS screening were observed at $m / z 348\left[\mathrm{M}+\mathrm{H}-\mathrm{H}_{2} \mathrm{O}\right]^{+}, 288[\mathrm{M}+\mathrm{H}$ $\left.-\mathrm{C}_{2} \mathrm{O}_{2} \mathrm{H}_{4}\right]^{+}, 228\left[\mathrm{M}+\mathrm{H}-\mathrm{C}_{4} \mathrm{O}_{4} \mathrm{H}_{8}\right]^{+}$in the positive ion spectrum. Based on MS and MS/MS data obtained and the analysis of elemental composition, the molecular formula of the ion was speculated of $\mathrm{C}_{20} \mathrm{H}_{31} \mathrm{NO}_{5}$. Combining with literature [24] it was inferred from acetyllycofawcine. Mass spectrum and proposed fragmentation pathway of acetyllycofawcine were shown in Figure 2. Finally, a total of 30 compounds were identified (characteristic peaks were labeled on the BPI chromatographic), including 21 alkaloids, 7 triterpenoids, and 2 other classes compound. The detailed information of compound name, molecular weight, molecular formula, and characteristic peak was shown in Table 1.

3.3. Multivariate Statistical Analysis and Identification of Plasma Sample after Oral Lycopodium clavatum In Vivo. In order to analyze the compounds of Lycopodium clavatum in plasma, the two groups were compared between the plasma sample of Lycopodium clavatum group and the control group that the chromatogram of BPI was shown in Figure 3, and then multivariate statistical analysis was used to further discover the prototype compounds in vivo.

PCA is a chemometric method that uses the idea of dimensionality reduction to transform multiple indicators into several comprehensive indicators with little information loss. In the PCA score (Figure 4), it could be observed that the plasma samples of drug-administered group and blank group were divided into two discrete groups, indicating that there was a significant difference in the drug-administered group and blank group. In order to further analyze the metabolic differences between the blank group and the drugadministered group in plasma samples, an in-depth analysis of the data (OPLS-DA) is required. According to the OPLSDA score (Figure 4), it was clearly observed that the two groups plasma samples were divided into 2 discrete groups, which was better than the score of PCA. VIP value reflects the contribution of the variable in the classification. The larger the VIP value, the greater the contribution of the variable to 
TABLE 1: Identification of the components from Lycopodium clavatum in vitro.

\begin{tabular}{|c|c|c|c|c|c|c|}
\hline NO. & $t_{\mathrm{R}}(\min )$ & Measured Mass $(\mathrm{m} / z)$ & Fragment ions $(m / z)$ & Formula & Error (ppm) & Identification \\
\hline 1 & 3.8002 & 278.1756 & $260,162,134$ & $\mathrm{C}_{16} \mathrm{H}_{23} \mathrm{NO}_{3}$ & 0 & $8 \beta$-hydroxylycoposerramine \\
\hline 2 & 4.1863 & 266.2119 & $248,230,202$ & $\mathrm{C}_{16} \mathrm{H}_{27} \mathrm{NO}_{2}$ & 0.38 & aeacetylfawcettiine \\
\hline 3 & 4.6209 & 292.1914 & $274,203,135$ & $\mathrm{C}_{17} \mathrm{H}_{25} \mathrm{NO}_{3}$ & 0.34 & palhinine A \\
\hline 4 & 4.6343 & 273.1963 & $243,200,186$ & $\mathrm{C}_{17} \mathrm{H}_{24} \mathrm{~N}_{2} \mathrm{O}$ & 1.46 & obscurinene \\
\hline 5 & 4.9963 & 296.185 & $278,260,234$ & $\mathrm{C}_{16} \mathrm{H}_{25} \mathrm{NO}_{4}$ & 4.05 & $4 \alpha, 8 \beta, 12 \beta$-trihydroxylycopodine \\
\hline 6 & 5.1711 & 274.1811 & $231,167,158$ & $\mathrm{C}_{17} \mathrm{H}_{23} \mathrm{NO}_{2}$ & 1.46 & lycojaponicumin $\mathrm{C}$ \\
\hline 7 & 5.2178 & 262.1807 & $234,206,164$ & $\mathrm{C}_{16} \mathrm{H}_{23} \mathrm{NO}_{2}$ & 0 & lycopoclavamine A \\
\hline 8 & 5.2195 & 260.1661 & $246,24,216$ & $\mathrm{C}_{16} \mathrm{H}_{21} \mathrm{NO}_{2}$ & 3.84 & huperzine E [4] \\
\hline 9 & 5.3981 & 280.1912 & $262,244,163$ & $\mathrm{C}_{16} \mathrm{H}_{25} \mathrm{NO}_{3}$ & 0.36 & $\begin{array}{c}6 \alpha, 8 \beta \text {-dihydroxylycopodine or } \\
4 \alpha, 8 \beta \text {-dihydroxylycopodine }\end{array}$ \\
\hline 10 & 5.5543 & 261.1966 & $245,202,188$ & $\mathrm{C}_{16} \mathrm{H}_{24} \mathrm{~N}_{2} \mathrm{O}$ & 0.38 & des-N-methyl- $\alpha$-obscurine \\
\hline 11 & 5.8878 & 275.2125 & $244,188,148$ & $\mathrm{C}_{17} \mathrm{H}_{26} \mathrm{~N}_{2} \mathrm{O}$ & 0.73 & $\alpha$-obscurine \\
\hline 12 & 6.0787 & 278.212 & $260,217,189$ & $\mathrm{C}_{17} \mathrm{H}_{27} \mathrm{NO}_{2}$ & 0 & (15R)-14,15-dihydroepilobscurinol \\
\hline 13 & 6.2025 & 248.2016 & $230,145,105$ & $\mathrm{C}_{16} \mathrm{H}_{25} \mathrm{NO}$ & 0.81 & lycopodine \\
\hline 14 & 6.4302 & 322.2037 & $244,216,176$ & $\mathrm{C}_{18} \mathrm{H}_{27} \mathrm{NO}_{4}$ & 5.9 & 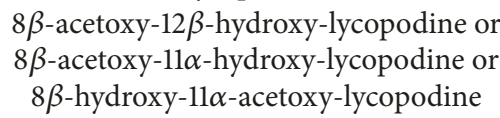 \\
\hline 15 & 6.4922 & 246.1854 & $218,176,124$ & $\mathrm{C}_{16} \mathrm{H}_{23} \mathrm{NO}$ & 1.62 & anhydrolycodoline \\
\hline 16 & 6.5863 & 264.1966 & $228,242,191$ & $\mathrm{C}_{16} \mathrm{H}_{25} \mathrm{NO}_{2}$ & 0.76 & lycodoline \\
\hline 17 & 6.7897 & 308.2226 & $248,230,159$ & $\mathrm{C}_{18} \mathrm{H}_{29} \mathrm{NO}_{3}$ & 0 & $\alpha$-lofoline \\
\hline 18 & 7.3916 & 276.1962 & $218,166,150$ & $\mathrm{C}_{17} \mathrm{H}_{25} \mathrm{NO}_{2}$ & 0.72 & lycoflexine \\
\hline 19 & 7.8795 & 366.2274 & $348,288,228$ & $\mathrm{C}_{20} \mathrm{H}_{31} \mathrm{NO}_{5}$ & 3.82 & acetyllycofawcine \\
\hline 20 & 8.2908 & 350.2324 & 290,230 & $\mathrm{C}_{20} \mathrm{H}_{31} \mathrm{NO}_{4}$ & 2.28 & acetylfawcettiine \\
\hline 21 & 8.5172 & 195.066 & $167,152,115$ & $\mathrm{C}_{10} \mathrm{H}_{10} \mathrm{O}_{4}$ & 1.54 & ferulic acid \\
\hline 22 & 10.2069 & 505.3513 & $487,263,199$ & $\mathrm{C}_{30} \mathrm{H}_{48} \mathrm{O}_{6}$ & 3.17 & 16-oxolyclanitin \\
\hline 23 & 11.0118 & 489.3571 & 471. 453,345 & $\mathrm{C}_{30} \mathrm{H}_{48} \mathrm{O}_{5}$ & 1.84 & japonicumin B or lycoclavanin \\
\hline 24 & 12.1919 & 473.362 & $102,111,167$ & $\mathrm{C}_{30} \mathrm{H}_{48} \mathrm{O}_{4}$ & 2.32 & $\begin{array}{c}3 R, 21 \alpha, 24 \text {-trihydroxyserrat-14-en- } \\
16 \text {-one }\end{array}$ \\
\hline 25 & 12.6387 & 475.377 & $457,410,237$ & $\mathrm{C}_{30} \mathrm{H}_{50} \mathrm{O}_{4}$ & 3.58 & Lycoclaninol [4] or japonicumin A \\
\hline 26 & 13.0973 & 461.362 & $443,441,102$ & $\mathrm{C}_{29} \mathrm{H}_{48} \mathrm{O}_{4}$ & 2.38 & lycojaponicuminol C \\
\hline 27 & 14.2839 & 445.3667 & $279,427,319$ & $\mathrm{C}_{29} \mathrm{H}_{48} \mathrm{O}_{3}$ & 3.37 & 26 -nor-8-oxo- $\alpha$-onocerin \\
\hline 28 & 15.3438 & 457.3664 & 439,397 & $\mathrm{C}_{30} \mathrm{H}_{48} \mathrm{O}_{3}$ & 3.93 & lycojaponicuminol A \\
\hline 29 & 15.5437 & 459.3828 & $441,398,397$ & $\mathrm{C}_{30} \mathrm{H}_{50} \mathrm{O}_{3}$ & 2.18 & $\begin{array}{c}\text { 3-epilycoclavanol or } \\
3 \beta, 21 \beta, 24-3 \text {-trihydroxyserrat-14-ene or } \\
3 \alpha, 21 \beta, 24 \text {-4-trihydroxyserrat-14-ene }\end{array}$ \\
\hline 30 & 16.1058 & $603.4045^{[\mathrm{M}-\mathrm{H}]}$ & $116,132,134$ & $\mathrm{C}_{39} \mathrm{H}_{56} \mathrm{O}_{5}$ & 0.66 & lycojaponicuminol D \\
\hline
\end{tabular}

the entire model classification. Generally, VIP value is greater than 1 as the characteristic variable of the metabolic. The value of VIP greater than 1 was screened and further analyzed. Finally, a total of 12 compounds were identified by the data of MS, MS/MS information, and the relevant literature and databases. They were lycojaponicumin C (6), huperzine E (8), des-N-methyl- $\alpha$-obscurine (10), $\alpha$-obscurine (11), lycopodine (13), $8 \beta$-acetoxy- $12 \beta$-hydroxy-lycopodine or $8 \beta$-acetoxy- $11 \alpha$ hydroxy-lycopodine or $8 \beta$-hydroxy-11 $\alpha$ - Acetoxylycopodine (14), lycodoline (16), $\alpha$-lofoline or fawcettiine (17), acetylfawcettiine (20), japonicumin B or lycoclavanin (23), $3 R, 21 \alpha$, 24-trihydroxyserrat-14-en-16-one (24) and lycojaponicuminol C (26). Among them, $\alpha$-obscurine (11) and lycodoline (16) were identified by comparison with the information of the standard, including retention time, MS and MSMS data. Next, the more standard will be purchased and used to further qualitative analysis of the compounds of Lycopodium clavatum.
3.4. Metabolites Identified Using MetaboLynx Tool. After the drug gets into the body, some components exist as the form of prototype, but most of them are structurally modified based on the original drug components, such as methylation and glutathione conjugation. The metabolite is structurally similar to the prototype drug and has characteristic mass spectrometry fragmentation of the prototype drug, so the structure of the metabolite can be inferred by characteristic fragment between the prototype and metabolite. Using MetaboLynx XS to process the data to analyze the plasma samples, most of the potential metabolites were identified. Finally, 5 metabolites were detected from the Lycopodium clavatum. The molecular weight of lycojaponicumin $\mathrm{C}$ (6) was 274.1811 and the formula was $\mathrm{C}_{17} \mathrm{H}_{23} \mathrm{NO}_{2}$. The precise molecular weight of M1 was 260.1653 that had a neutral loss of $14 \mathrm{Da}$ compared with lycojaponicumin C (6) and the main fragment ions was 167,158 , and 125 that were the same as the main fragment ions of lycojaponicumin C (6); it is inferred that M1 


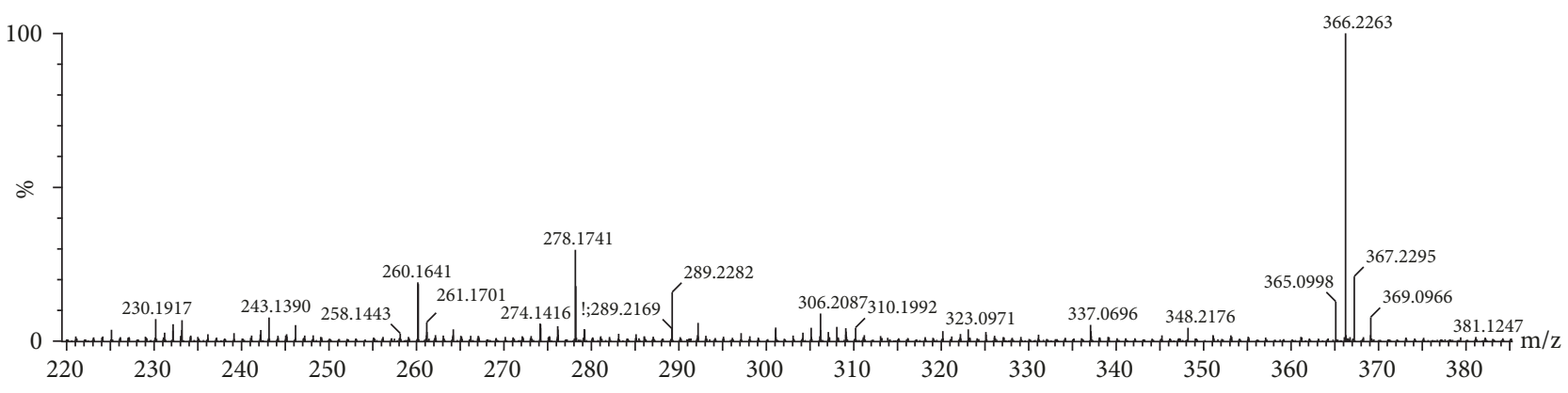

(a)

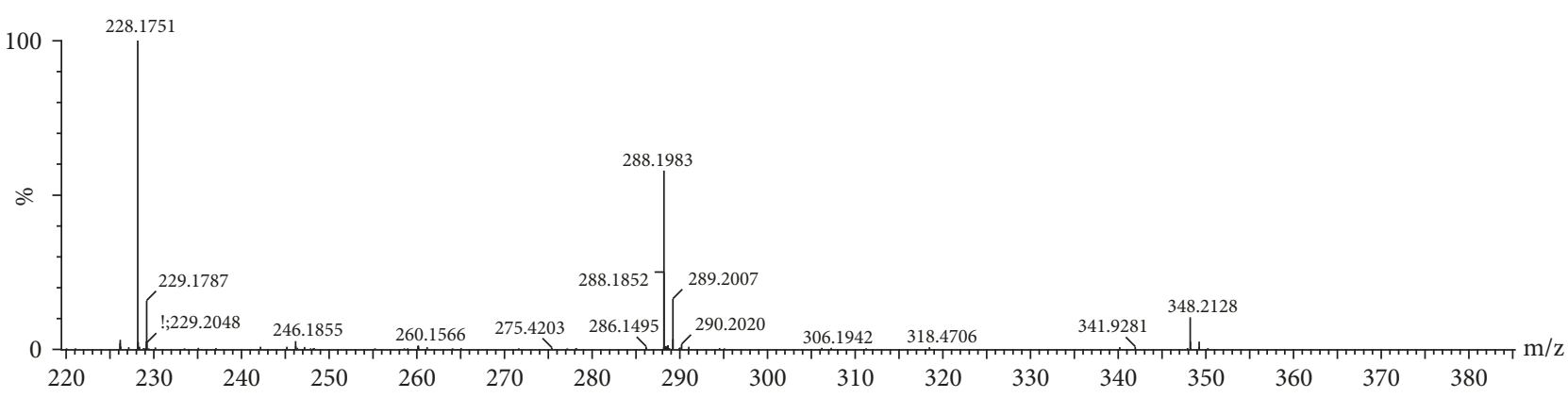

(b)

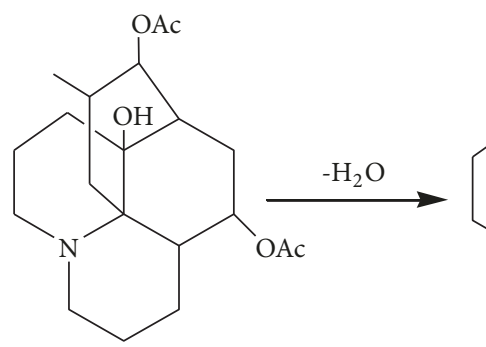

$m / z=366$
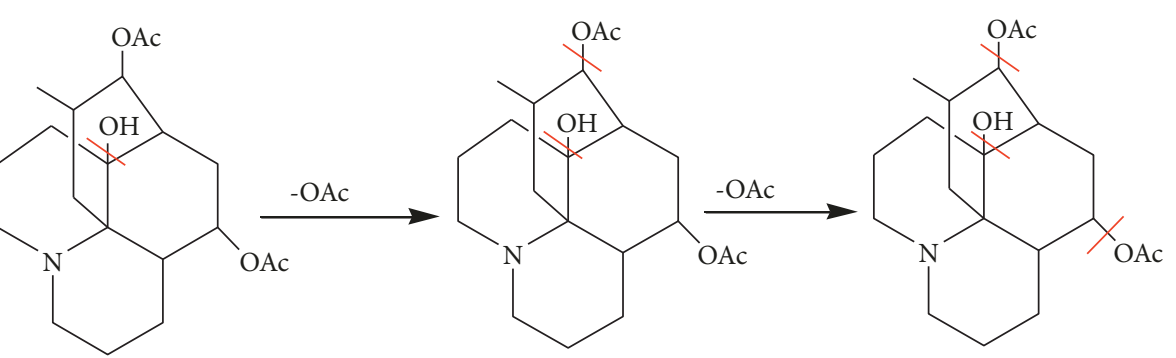

$m / z=348$
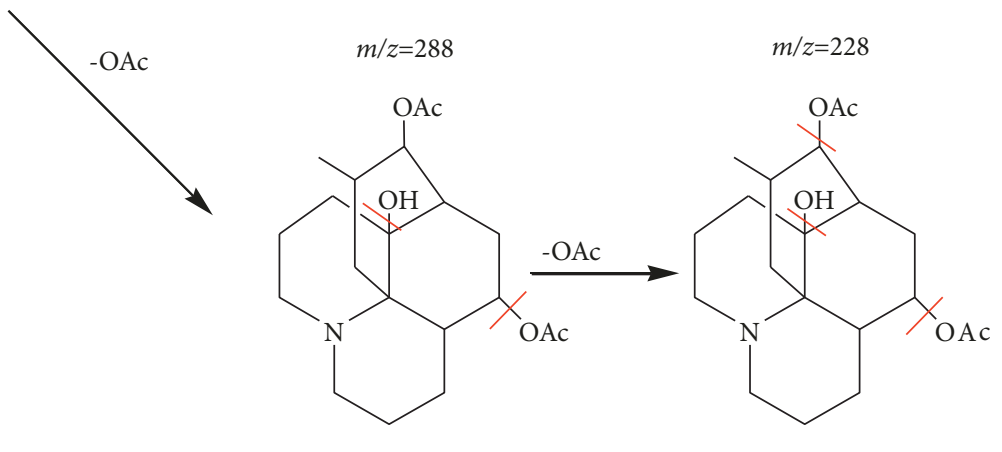

$m / z=288$

$m / z=228$

(c)

FIGURE 2: The mass spectra of acetyllycofawcine analyzed by UHPLC-Q-TOF-MS. the MS spectrum (a); the MSMS spectrum (b); proposed fragmentation pathways of acetyllycofawcine (c).

is a metabolite of the demethylation of the lycojaponicumin C. Finally, a total of 5 metabolites were identified the same way. M2 is a metabolite of the methylation of the palhinine A, M3 is a metabolite of the methylation of the (15R)-14,15dihydroepilobscurinol, M4 is a metabolite of the methylation of lycodoline, and M5 is a metabolite of the demethylation of $\alpha$-lofoline. The detailed information of prototype, metabolite name, molecular weight, and molecular formula was given in Table 2.

\section{Conclusion}

In this work, we demonstrated a systematic and reliable analytical method using UPLC-Q-TOF-MS coupled with multiple data processing approaches for rapidly screening and identifying the chemical constituents of Lycopodium clavatum both in vitro and in vivo. In vitro, 30 peaks were characterized in $75 \%$ ethanol extract of Lycopodium clavatum. In vivo, 17 peaks were characterized in rat plasma 


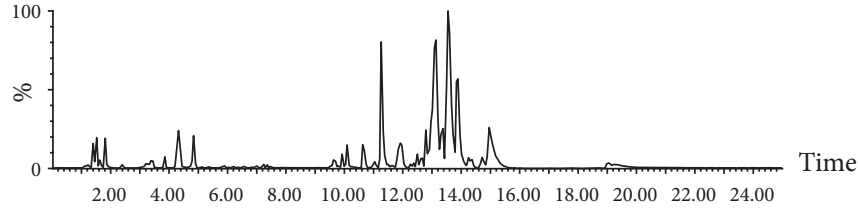

(a)

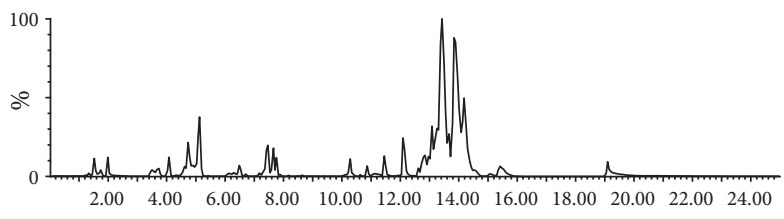

(c)

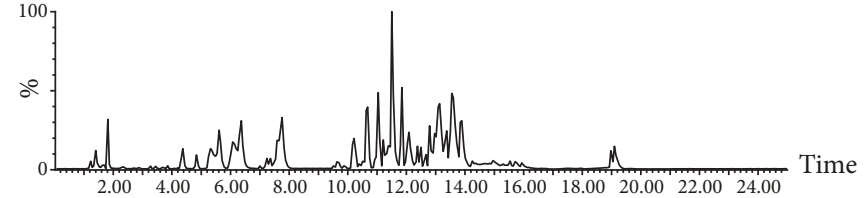

(b)

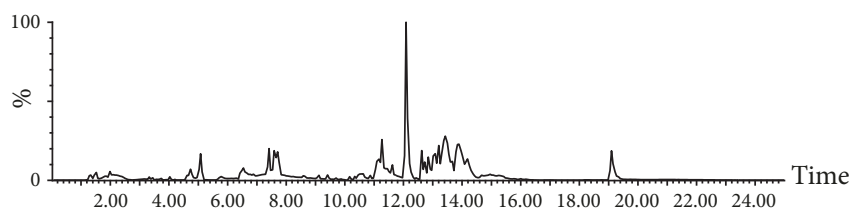

(d)

FIGURE 3: ESI base peak ion (BPI) chromatogram of the plasma sample of Lycopodium clavatum extracts analyzed by UPLC-Q-TOF/MS in both positive (a) and negative (b) ion mode; ESI base peak ion (BPI) chromatogram of the control plasma sample of Lycopodium clavatum extracts analyzed by UPLC-Q-TOF/MS in both positive (c) and negative (d) ion mode.

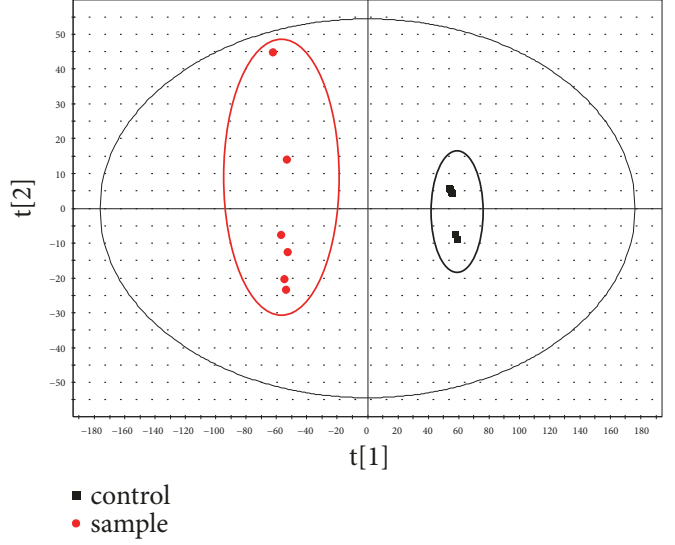

(a)

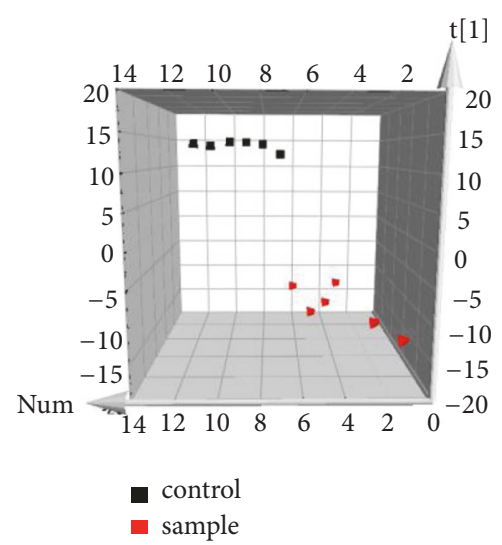

(c)

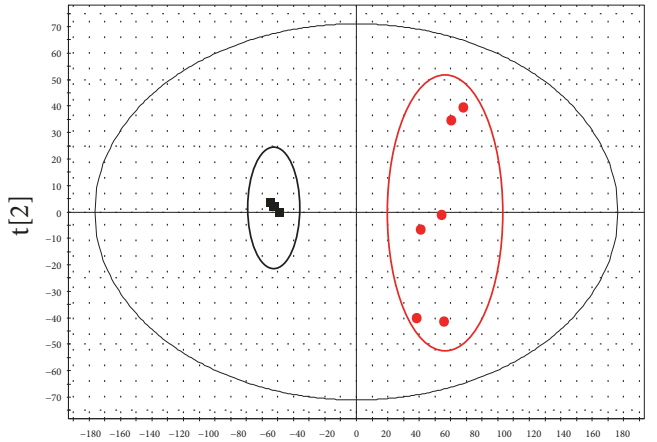

$\mathrm{t}[1]$

- control

- sample

(b)

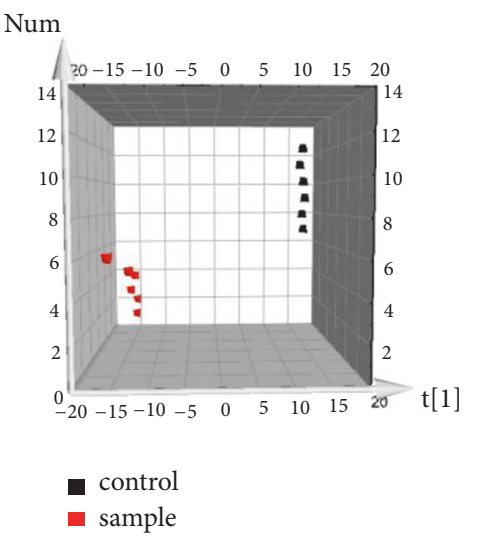

(d)

FIGURE 4: Multivariate statistical analysis and identification of plasma sample of Lycopodium clavatum. PCA of Lycopodium clavatum group vs. control group in positive mode (a) and negative mode (b); 3D-OPLS-DA of Lycopodium clavatum group vs. control group in positive mode (c) and negative mode $(\mathrm{d})$. 
TABLE 2: Identification of the metabolites from Lycopodium clavatum in vivo.

\begin{tabular}{lcccccc}
\hline NO. & $\mathrm{t}_{\mathrm{R}}(\mathrm{min})$ & Measured Mass $(\mathrm{m} / z)$ & Metabolite Name & Formula & Error (ppm) & Prototype \\
\hline M1 & 5.2 & 260.1653 & demethylation & $\mathrm{C}_{16} \mathrm{H}_{21} \mathrm{NO}_{2}$ & 1 & lycojaponicumin C \\
M2 & 6.95 & 306.2077 & methylation & $\mathrm{C}_{18} \mathrm{H}_{27} \mathrm{NO}_{3}$ & 2.6 & palhinine A \\
M3 & 9.17 & 292.2287 & methylation & $\mathrm{C}_{18} \mathrm{H}_{29} \mathrm{NO}_{2}$ & 3.7 & (15R)-14,15-dihydroepilobscurinol \\
M4 & 7.12 & 278.2127 & methylation & $\mathrm{C}_{17} \mathrm{H}_{27} \mathrm{NO}_{2}$ & 2.6 & lycodoline \\
M5 & 3.57 & 294.2076 & demethylation & $\mathrm{C}_{17} \mathrm{H}_{27} \mathrm{NO}_{3}$ & 2.4 & $\alpha$-lofoline \\
\hline
\end{tabular}

that including 12 prototype compounds and 5 metabolites. Methylation and demethylation are the main transformation reactions of Lycopodium clavatum in rat plasma. This work could be helpful to understand the complex compounds of Lycopodium clavatum and further analyze the pharmacological studies of active compounds.

\section{Data Availability}

The data used to support the findings of this study are included within the article.

\section{Conflicts of Interest}

The authors declare no conflicts of interest in the paper.

\section{Authors' Contributions}

Xin Li and Mingqin Kang as co-first authors contributed equally to the paper.

\section{Acknowledgments}

This work received the support of grants from the National Natural Science Foundation of China [grant numbers 8180141249], Tianjin Education Commission Research Project in China [grant numbers 2017KJ132, 2017KJ153], and Scientific and Technological Project of General Administration of Quality Supervision, Inspection and Quanrantine of the Peoples Republic of China [2012IK 162 and 2013IK 162].

\section{Supplementary Materials}

Table S1: The database of 121 prototype compounds of Lycopodium clavatum. (Supplementary Materials)

\section{References}

[1] I. Orhan, E. Küpeli, B. Şener, and E. Yesilada, "Appraisal of antiinflammatory potential of the clubmoss, Lycopodium clavatum L.," Journal of Ethnopharmacology, vol. 109, no. 1, pp. 146-150, 2007.

[2] I. Orhan, B. Özçelik, S. Aslan et al., "Antioxidant and antimicrobial actions of the clubmoss Lycopodium clavatum L.," Phytochemistry Reviews, vol. 6, no. 1, pp. 189-196, 2007.

[3] I. Orhan, S. Terzioglu, and B. Şener, " $\alpha$-Onocerin: an acetylcholinesterase inhibitor from Lycopodium clavatum," Planta Medica, vol. 69, no. 3, pp. 265-267, 2003.
[4] I. Orhan, B. Şener, M. I. Choudhary, and A. Khalid, "Acetylcholinesterase and butyrylcholinesterase inhibitory activity of some Turkish medicinal plants," Journal of Ethnopharmacology, vol. 91, no. 1, pp. 57-60, 2004.

[5] J. M. Rollinger, J. Ewelt, C. Seger, S. Sturm, E. P. Ellmerer, and H. Stuppner, "New insights into the acetylcholinesterase inhibitory activity of Lycopodium clavatum," Planta Medica, vol. 71, no. 11, pp. 1040-1043, 2005.

[6] R. Gebhardt, "Antioxidative, antiproliferative and biochemical effects in HepG2 cells of a homeopathic remedy and its constituent plant tinctures tested separately or in combination," Drug Research, vol. 53, no. 12, pp. 823-830, 2003.

[7] X. Wang, G. Zhang, P. Zhuang et al., "Lycojaponicumins A-C, three alkaloids with an unprecedented skeleton from Lycopodium japonicum," Organic Letters, vol. 14, no. 10, pp. 2614-2617, 2012.

[8] X.-L. Li, Y. Zhao, X. Cheng et al., "Japonicumins A-D: four new compounds from Lycopodium japonicum," Helvetica Chimica Acta, vol. 89, no. 7, pp. 1467-1473, 2006.

[9] J. Yan, X. Zhang, Z. Li et al., "Three new triterpenoids from Lycopodium japonicum thunb," Helvetica Chimica Acta, vol. 88, no. 2, pp. 240-244, 2005.

[10] Z. Zhang, H. N. ElSohly, M. R. Jacob, D. S. Pasco, L. A. Walker, and A. M. Clark, "Natural products inhibiting Candida albicans secreted aspartic proteases from Lycopodium cernuum," Journal of Natural Products, vol. 65, no. 7, pp. 979-985, 2002.

[11] Y. Zhang, P. Yi, Y. Chen, Z. Mei, X. Hu, and G. Yang, "Lycojaponicuminol A-F: cytotoxic serratene triterpenoids from Lycopodium japonicum," Fitoterapia, vol. 96, pp. 95-102, 2014.

[12] Y. Hirasawa, J. Kobayashi, and H. Morita, "The Lycopodium alkaloids," Heterocycles, vol. 77, no. 2, pp. 679-729, 2009.

[13] X. Ma and D. R. Gang, "The Lycopodium alkaloids," Natural Product Reports, vol. 21, no. 6, pp. 752-772, 2004.

[14] W. Cao, X. Wang, H. Li et al., "Studies on metabolism of total glucosides of paeony from Paeoniae Radix Alba in rats by UPLC-Q-TOF-MS/MS," Biomedical Chromatography, vol. 29, no. 11, pp. 1769-1779, 2015.

[15] H. Ma, Y. Liu, X. Mai et al., "Identification of the constituents and metabolites in rat plasma after oral administration of Huanglian Shangqing pills by ultra high-performance liquid chromatography/quadrupole time-of-flight mass spectrometry," Journal of Pharmaceutical and Biomedical Analysis, vol. 125, pp. 194-204, 2016.

[16] F. Zhu, J. Chen, H. Wang et al., "Analysis of the chemical constituents and rats metabolites after oral administration of Nauclea officinalis by ultra-performance liquid chromatography quadrupole time-of-flight mass spectrometry," Journal of Chromatography B, vol. 1007, pp. 54-66, 2015.

[17] Y. Lecompte, M. Rosset, C. Richeval, L. Humbert, and P. Arpino, "UPLC-ESI-Q-TOF-MSE identification of urinary metabolites of the emerging sport nutrition supplement methoxyisoflavone 
in human subjects," Journal of Pharmaceutical and Biomedical Analysis, vol. 96, pp. 127-134, 2014.

[18] Y. Yang, X. J. Zhao, Y. Pan, and Z. Zhou, "Identification of the chemical compositions of Ponkan peel by ultra performance liquid chromatography coupled with quadrupole time-of-flight mass spectrometry," Analytical Methods, vol. 8, no. 4, pp. 893903, 2016.

[19] H. Wang, C. Chen, Y. Liu, X. Yang, and H. Xiao, "Metabolic profile of Yi-Xin-Shu capsule in rat by ultra-performance liquid chromatography coupled with quadrupole time-of-flight tandem mass spectrometry analysis," RSC Advances, vol. 5, no. 98, pp. 80583-80590, 2015.

[20] Y. Sun, J. Yan, H. Meng et al., "A new alkaloid from Lycopodium japonicum Thunb," Helvetica Chimica Acta, vol. 91, no. 11, pp. 2107-2109, 2008.

[21] J. He, X. Chen, M. Li et al., "Lycojapodine A, a novel alkaloid from Lycopodium japonicum," Organic Letters, vol. 11, no. 6, pp. 1397-1400, 2009.

[22] B. Li, W. Zhang, Y. He, L. Lu, D. Kong, and Y. Shen, "New alkaloids from Lycopodium japonicum," Chemical \& Pharmaceutical Bulletin, vol. 60, no. 11, pp. 1448-1452, 2012.

[23] X.-J. Wang, L. Li, S.-S. Yu et al., "Five new fawcettimine-related alkaloids from Lycopodium japonicum Thunb," Fitoterapia, vol. 91, pp. 74-81, 2013.

[24] X.-J. Wang, L. Li, Y.-K. Si et al., "Nine new lycopodine-type alkaloids from Lycopodium japonicum Thunb," Tetrahedron, vol. 69, no. 30, pp. 6234-6240, 2013. 


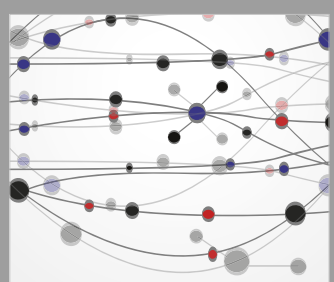

The Scientific World Journal
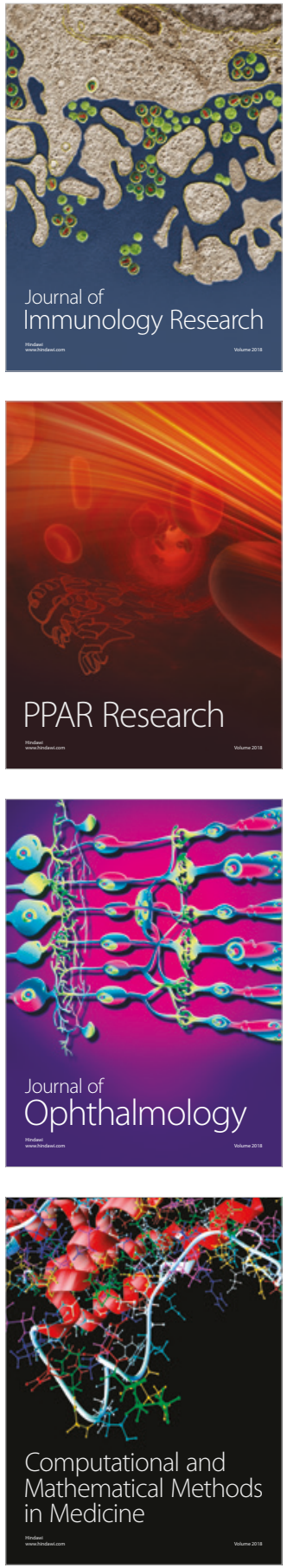

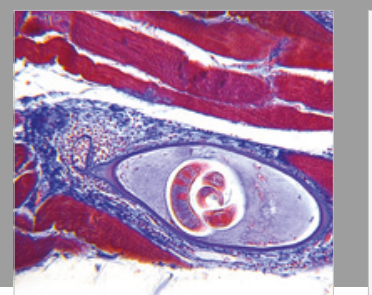

Gastroenterology Research and Practice

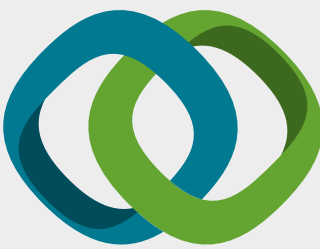

\section{Hindawi}

Submit your manuscripts at

www.hindawi.com
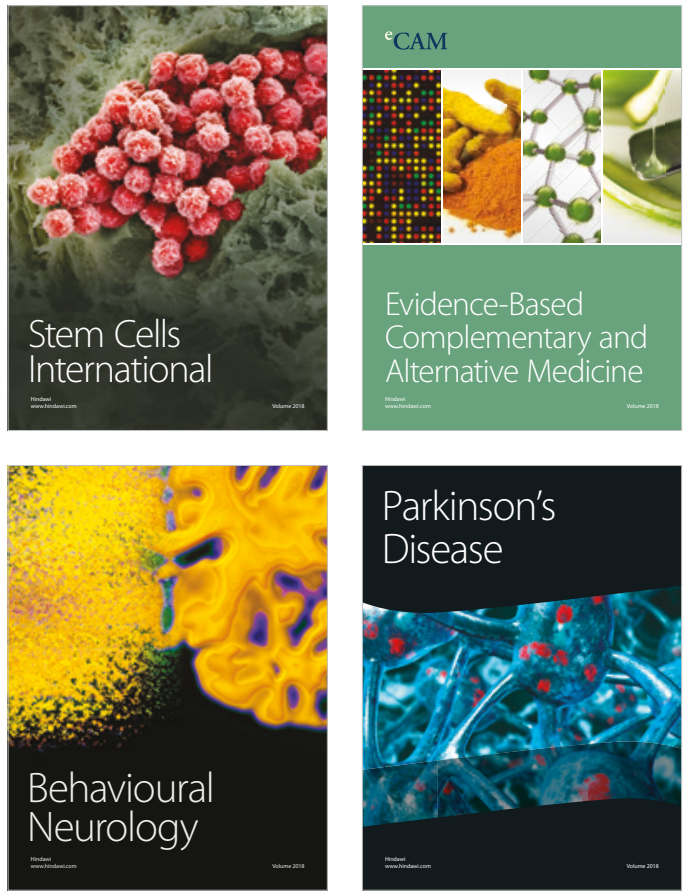

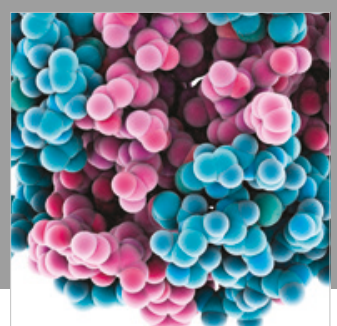

ournal of

Diabetes Research

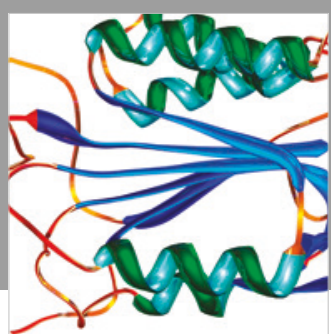

Disease Markers
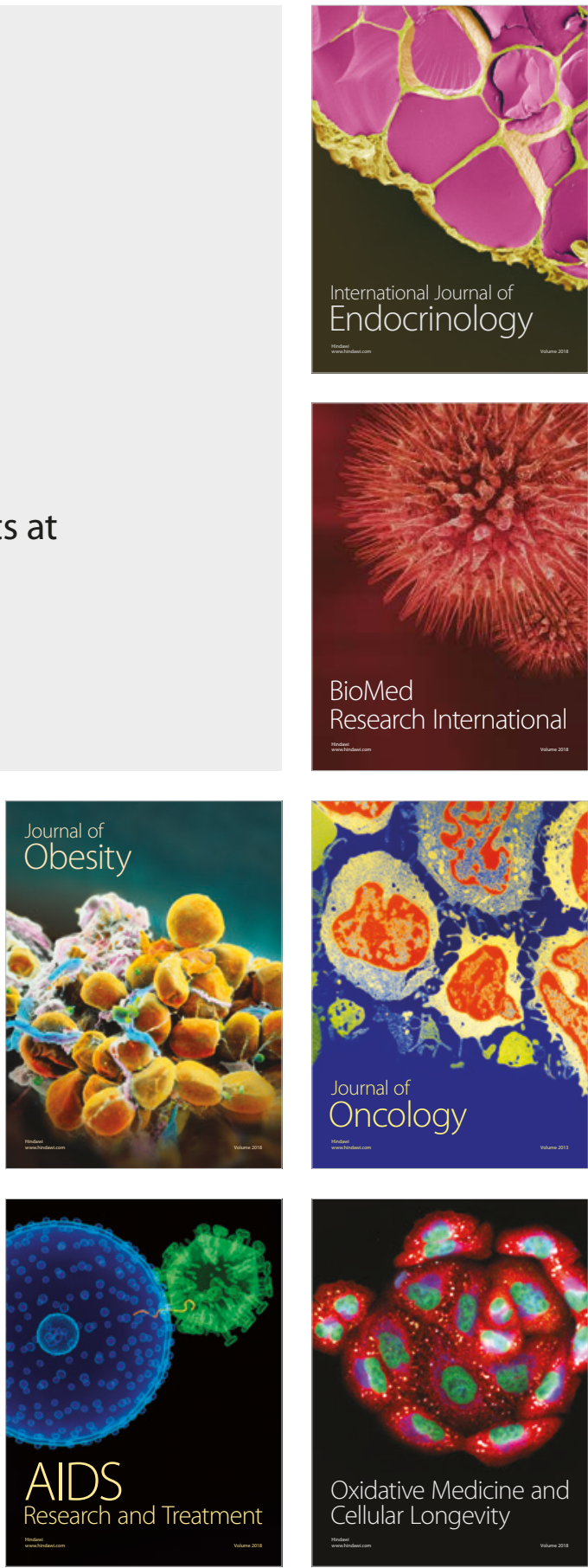